determine the need for topical glucocorticoid therapy (TGC) in patients with JIA-U on systemic biological therapy in comparison to patients treated with methotrexate (MTX) only.

We have conducted longitudinal observational study with JIA-U patients in whom systemic immunomodulatory treatment (IMT: biologics and/or MTX) was introduced and who were followed at least 3 months in the period between 2011 and 2017. The data about the number of cells in the anterior chamber (AC) according to Standardization of Uveitis Nomenclature (SUN) Working Group criteria, about TGC and systemic therapy and JIA complications were collected on each examination. Generalized linear mixed models were used to analyze the relationships between treatment with biologics, MTX, TGC and the grade of inflammation in AC according to SUN criteria.

38 JIA-U patients (69 eyes) with median (range) age of 4.9 (2-15) years and follow up period of 209 (19-381) weeks were included. There were a total of 1205 examinations. At the first examination JIA-U was detected in $16(42.1 \%)$ of patients, $59(79.7 \%)$ of the eyes had $\leq 1+$ cells in the AC, and in $19(50 \%)$ of JIA-U patients complications were already present. MTX was introduced in 23 (60.5\%) JIA-U patients before the inclusion in the study, $8(21 \%)$ has already received biologics, while in $4(10.5 \%)$ prior systemic glucocorticoids were also used. Until the end of the study, all patients received MTX and 40\% JIA-U were treated with biologics. The average number of TGC doses decreased from 3.74 at baseline to 0.72 in the 48 th month.

After Friedman and the post hoc test a significant difference in the daily doses of TGC could be seen from the 12th month after application of systemic IMT. The number of daily doses of TGC as well as the degree of inflammation in AC per eye decreased over time. Using generalized linear mixed models it was shown that the treatment with biologics, but not with MTX and systemic glucocorticoids, was associated with lower intensity of TGC therapy.

Treatment with biologics and systemic glucocorticoids, but not with MTX, were associated with lower degree of inflammation in AC.

The results showed that the application of systemic biological therapy may result in less intensive TGC therapy, resulting in glucocorticoid-sparing potential, and reducing intraocular inflammation.

\section{DETERMINING THE EFFECTIVENESS OF SYSTEMIC IMMUNOMODULATORY THERAPY IN THE TREATMENT OF PATIENTS WITH JUVENILE IDIOPATHIC ARTHRITIS ASSOCIATED UVEITIS DEPENDING ON THE CHOSEN OUTCOME MEASURES}

Barisic Kutija Marija, Sestan Mario*, Peric Sanja, Kifer Nastasia, Ivkic Petra Kristina, Galiot Delic Martina, Knezevic Josip, Held Martina, Frkovic Marijan, Jelusic Marija, Vukojevic Nenad. University Hospital Centre Zagreb, Department of Paediatrics, University of Zagreb School of Medicine

\subsection{6/archdischild-2021-europaediatrics.445}

The number of patients with juvenile idiopathic arthritis associated uveitis (JIA-U) who need systemic immunomodulatory treatment (IMT) for disease control is small. Variabilities in the patient selection and the results on the effectiveness of IMT make it difficult to compare studies. We aimed to show on the same sample of JIA-U patients how different the obtained levels of therapy efficacy are depending on the selected outcomes.

The longitudinal observational study with JIA-U patients treated with IMT was conducted at University Hospital Centre Zagreb in the period from 2011 to 2017.

We included 38 JIA-U patients aged 2 to 15 years and 69 eyes (7 patients had unilateral JIA-U). At baseline 46 (66.7\%) eyes had grade $\leq 1+$ of inflammation in anterior chamber (AC) according to Standardization of Uveitis Nomenclature (SUN) Working Group criteria, 11 (15.9\%) had grade 2+, 3 (4.4\%) had grade $3+.23$ children $(60.5 \%)$ had already received methotrexate (MTX), 8 (21.0\%) biologics, 4 $(10.5 \%)$ systemic glucocorticoids (GC). Topical glucocorticoids (TGC) in the form of drops were used in $61(88.4 \%)$ eyes with a median of 4 daily doses, GC ointment in $75.4 \%$ of the eyes with a median of 1 dose. Until the end of the follow-up, all children received MTX, and 40\% of them biologics. The results of the effectiveness of IMT are presented according to the reduction of the need for TGC and achieved level of inflammation in AC. In the first 12 months, among JIA-U patients treated with both biologics and MTX, in $65 \%$ of eyes there was no need for TGC therapy, in the 48th month in 50\% of eyes, while the rest required 1-2 daily doses of TGC. At the end of the first year, with MTX and biological therapy $75 \%$ of eyes had grade 0 of inflammation and in 48th month $61.1 \%$. In the 12 th month in $75 \%$ of eyes a grade 0 of inflammation was achieved with $\leq 2$ doses of TGC, and in the 48th month in $61.1 \%$. According to milder criteria, in the 12th month $90 \%$ of the eyes have a degree of inflammation $\leq 0,5+$ with $\leq 2$ doses of TGC, and in the 48th month all patients achieved this goal.

It was shown that the results of treatment outcomes during follow-up largely depend on the selected outcome measures. This will be important for future research because setting different limits can lead to a more favorable outcome.

\section{SINGLE NUCLEOTIDE POLYMORPHISMS OF GENES HMGB1 AND AGER AND ITS ASSOCIATION WITH CLINICAL FEATURES OF IGA VASCULITIS}

Martina Held*, Mateja Batnozic Varga, Mario Sestan, Matej Sapina, Nastasia Kifer, Danica Grguric, Kristina Crkvenac Gornik, Marijan Frkovic, Nena Arvaj, Jasenka Wagner, Marija Jelusic. University Hospital Centre Zagreb, University of Zagreb School of Medicine

\subsection{6/archdischild-2021-europaediatrics.446}

IgA vasculitis (IgAV) is a desease in which genetic background also plays an important role. Some small studies have indicated the importance of variants in various non-HLA genes in the manifestation of different disease phenotypes. The aim of this research was to investigate single nucleotide polymorphisms (SNPs) of genes HMGB1 and AGER encoding for high mobility group box-1 (HMGB1) and receptor for advanced glycation endproducts (RAGE), both acting as mediators of inflammation, in the susceptibility and clinical features of patients with $\mathrm{IgAV}$.

HMGB1 and RAGE gene polymorphisms were genotyped using a real-time polymerase chain reaction. The presence and frequency of polymorphisms in HMGB1 (rs2249825, rs1045411, rs1060348, rs1412125 and rs41369348) and RAGE (rs1800625, rs1800624, rs2070600 and rs3134940) were determined. Clinical data were collected from database 
of IgAV patients from two Croatian University Centers for pediatric rheumatology.

81 pediatric IgAV patients were included, of whom 45 were boys and 36 girls, as well as 150 age- and sex-matched healthy controls without any history of autoimmune disease. The median (range) age of IgAV patients was 6.25 (4.60-8.20) years, and among them $71.6 \%$ had joint involvement, $29.62 \%$ had gastrointestinal manifestations, while $27.16 \%$ developed nephritis. The purpuric rash which extended from lower extremities to the trunk, upper extremities and face (generalized rash) was present in $43.20 \%$ of patients and $27.16 \%$ had at least one relapse. Among the analyzed polymorphisms, only in the rs1412125 there was a deviation from the Hardy Weinberger equilibrium. There was no statistically significant association of the analyzed polymorphisms with the IgAV susceptibility, compared to healthy controls. Polymorphism rs2070600 was significantly related with the development of nephritis in IgAV, while rs1412125 was associated with gastrointestinal involvement. The IgAV patients carrying the $\mathrm{T}$ allele (rs2070600) of the AGER had significantly increased risk of nephritis development compared with the IgAV patients with homozygous CC genotype in dominant (OR 4.05, CI 1.0915.03, $\mathrm{p}=0.037$ ) and additive genetic models (OR 3.95, CI 1.16-13.47, $\mathrm{p}=0.049$ ). The minor $\mathrm{C}$ allele (rs1412125) of the HMGB1 was found to significantly increase the risk of gastrointestinal involvement in overdominant model with an allelic odd ratio of 2.78 (CI 1.04-7.43, p $=0.04$ ).

Although neither of analyzed HMGB1 and RAGE polymorphisms was not associated with IgAV susceptibility, our results indicated that these polymorphisms may be involved in the pathogenesis of IgAV with possible effect on different disease phenotypes.

SUPPORT: Croatian Science Foundation project IP-2019-048822.

\section{SYSTEMIC JUVENILE IDIOPATHIC ARTHRITIS WITHOUT ARTHRITIS AS THE CAUSE OF INTERMITTENT PERSISTENT FEVER IN A 7-YEAR OLD GIRL}

${ }^{1}$ Ana Smajo*, 'Vana Vukić, ${ }^{1,2}$ Lovro Lamot. 'University of Zagreb, School of Medicine; ${ }^{2}$ Division of Clinical Immunology and Rheumatology, Department of Pediatrics, Clinical Hospital Center Sestre Milosrdnice, Zagreb, Hrvatska

10.1136/archdischild-2021-europaediatrics.447

Background Intermittent fever along with increased inflammatory markers (CRP and ESR) most commonly is the sign of an infectious disease. Nevertheless, without an adequate response to antibiotic treatment, it should raise concern of other conditions, including several rheumatic, like Kawasaki disease, periodic fever syndromes and systemic form of juvenile idiopathic arthritis (JIA), even when no clear arthritis is present.

Case Presentation We present a case of a 7-year-old girl with a 14-month history of intermittent fever with increased inflammatory markers, along with unspecific symptoms such as hepatomegaly, polymorphous rash and migratory arthralgia. An extensive diagnostic workup excluded infectious aetiology, genetic testing did not detect pathogenic mutations. Despite the treatment with intravenous imunoglobulines and low dose glucocorticoids (GCs), the fever did not subside. Finally, extensive laboratory workup revealed increased proinflammatory cytokines IL-6 and TNF-alpha along with chronic anaemia and thrombocytosis. The systemic subtype of JIA was considered, and treatment with pulse $(30 \mathrm{mg} / \mathrm{kg})$, continued with high $(2 \mathrm{mg} / \mathrm{kg})$ doses of GCs was initiated with an instant resolution of symptoms. Nevertheless, after the weaning of GCs, the new exacerbation was observed and therefore tocilizumab, humanized monoclonal antibody against IL-6 receptor, was initiated.

Conclusion Systemic form of juvenile idiopathic arthritis is a heterogeneous disease dominated musculoskeletal and systemic symptoms. While the former ones are a result of similar pathophysiologiocal mechanisms as other forms of JIA, activation of the nonspecific immune response is responsible for the sytemic ones, similar to other autoinflammatory diseases. Therefore, it is possible that in some cases of systemic form of JIA, systemic inflammation is present without the musculoskeletal symptoms. With the existing classification criteria of the International League against rheumatism (ILAR) it can be difficult to diagnose and initiate appropriate therapy. Consequently it can mislead to recognize a serious complication macrophage activation the syndrome. The recognition of the unique nature of systemic JIA in comparison to other types of JIA as well as an increased understanding of its pathogenesis, provides a better outcome and prognosis for children who often go undiagnosed with a debilitating chronic condition.

\section{\begin{tabular}{|l|l}
\hline 448 & TREATMENT OF JUVENILE SPONDYLOARTHRITIS
\end{tabular}}

${ }^{1}$ Vana Vukić ${ }^{*},{ }^{2}$ Mandica Vidović, ${ }^{1,2}$ Miroslav Harjaček, ${ }^{1,2}$ Lovro Lamot. ${ }^{1}$ School of Medicine University of Zagreb; ${ }^{2}$ Department of Clinical Immunology and Rheumatology, Department of Pediatrics, University Hospital Centre Sestre milosrdnice, Zagreb, Croatia

10.1136/archdischild-2021-europaediatrics.448

Juvenile spondyloarthritis (jSpA) represents a spectrum of inflammatory arthritis with strong HLA-B27 association and involvement of enthesis and/or axial skeleton that appears in children and young adults. ILAR classification criteria for enthesitis related arthritis subtype of juvenile idiopathic arthritis, which is undifferentiated form of jSpA, includes arthritis, enthesitis, presence of sacroiliac joint tenderness, inflammatory lumbosacral pain, HLA-B27 positivity, positive family history and acute anterior uveitis.

We present a case of 16 years old girl diagnosed with juvenile spondyloarthritis that first presented with recurrent monthly swelling of index finger at the age of 14 . Symptoms progressed to low back pain in the morning which partly declined with activity. Additionally, her right knee was swollen and painful. She was examined on multiple occasions by pediatric orthopedic surgeon and in pediatric emergency department before seeing a pediatric rheumatologist. The first examination revealed sacroiliac joint tenderness with positive FABER test and abnormal modified Schober test. Family history was negative for rheumatic diseases and there were no signs of uveitis nor enthesitis, with ANA, RF and extensive laboratory workup being either negative or within reference range. However, HLA-B27 turned positive and MRI showed right sacroiliitis. NSAID was prescribed but symptoms nevertheless persisted. Thus, after the exclusion of TBC with quantiferon test, intraarticular corticosteroid injection was applied to the right knee and oral corticosteroid was introduced as 